\title{
Accès à la grand-parentalité et remaniements psychiques au Rorschach : à propos d'un cas clinique
}

\author{
Margaux Bouteloup, Rose-Angélique Belot et André Mariage
}

\section{Introduction}

Le rôle des grands-parents est régulièrement étudié comme soutien dans l'accès à la parentalité (Belot et coll., 2013; Vennat, Panagiotou et Mellier, 2018) et dans le développement de l'enfant (Soulé, 1979; Miljkovitch, 2005). L'inverse, c'est-à-dire les modifications subjectives et les bénéfices du rôle de grand-parent, n'est quant à lui que peu abordé dans la littérature, qui traite majoritairement des aspects sociologiques en jeu.

\section{La place du grand-parent dans la société}

Il y a encore une cinquantaine d'années, les personnes âgées pouvaient être considérées comme une «bouche supplémentaire à nourrir» (Attias-Donfus, 2008). En quelques générations, les grands-parents sont passés du statut de «charge » à celui de support familial pouvant assurer au quotidien un véritable relais pour certaines fonctions parentales (garde des enfants, disponibilité, tendresse, transmission de valeurs...). Le contexte sociodémographique a participé à faire évoluer ce statut : allongement de la durée de vie, recul de l'âge de la première naissance qui repousse l'âge de la grand-parentalité, départ à la retraite de plus en plus tardif... Autant de facteurs qui redessinent progressivement les contours de la grandparentalité et ses fonctions.

Marie-Claude Mietkiewicz et Benoît Schneider (2010) retracent à partir de l'analyse de la littérature jeunesse les nouveaux rôles et places attribués aux grands-parents. Dans les histoires destinées aux enfants, les grands-parents sont racontés à travers les relations interpersonnelles familiales qu'ils entretiennent avec leurs petits-enfants. Cependant, ils ne semblent exister qu'à travers leurs rôles grand-parentaux et leur vie sociale ou amicale n'apparaît pas. Les relations avec la génération «intermédiaire » sont quasi absentes, voire disqualifiées et exclues. Serge Lebovici (1991, p. 182) nous rappelle pourtant que «la fonction des grands-parents devrait être simple : aimer les petits-enfants et aider les parents ». Or, il apparaît que dans la littérature jeunesse la notion d'assistance des grands-parents à leurs enfants, de soutien au rôle parental, disparaît progressivement au profit d'une relation élective et réciproque entre la première et la troisième génération, relation au sein de laquelle sont prônées des valeurs de «soutien réciproque qui fonde la complicité et renforce l'alliance intergénérationnelle» (Mietkiewicz et Schneider, 2010, p. 90). Ceci est révélateur de l'évolution sociétale génératrice de nouveaux rôles des grands-parents qui doivent tout à la fois trouver «la juste place » et la «bonne distance» avec leur enfant et leur petit-enfant (Mietkiewicz et Schneider, 2013).

Margaux Bouteloup, laboratoire de psychologie EA 3188, UFR SLHS, université Bourgogne Franche-Comté ; service de neurologie, centre hospitalier régional universitaire de Besançon. margaux.bouteloup@live.fr

Rose-Angélique Belot, laboratoire de psychologie EA 3188, UFR SLHS, université Bourgogne Franche-Comté. rose-angelique.belot@univ-fcomte.fr

André Mariage, laboratoire de psychologie EA 3188, UFR SLHS, université Bourgogne Franche-Comté. andre.mariage@univ-fcomte.fr 


\section{Grand-parenté, grand-parentalité, grand-maternité : quel vécu psychique?}

La grand-parenté se distingue de la grand-parentalité en ce qu'elle correspond uniquement à un statut auquel le sujet est assigné : le grand-parent le deviendra, indépendamment de son désir, si son enfant décide d'être parent et au moment où ce dernier le souhaite. Il est alors seulement question d'un choix du couple, avec lequel chaque grand-parent devra apprendre à composer. La grand-parentalité, elle, s'érige sur la volonté du grand-parent à prendre ce rôle. Il s'agit d'une construction au sein d'une filiation où le sujet va devoir transformer ses rapports à lui-même, à son enfant et à la société pour créer une relation avec ce petit-enfant né ou à venir, dans une période de sa vie où il peut parfois être difficile d'accepter de se voir vieillir (Schneider, Mietkiewicz et Bouyer, 2005). La création d'une nouvelle dynamique de relation avec le petit-enfant représente également un enjeu psychique important pour le grandparent: son engagement ou son retrait dans ses fonctions grand-parentales dépendront notamment de son parcours de vie (Le Borgne-Uguen, 2003), du rapport à son enfant devenu parent (Thévenot, 2005) mais aussi de son rapport à ses propres grands-parents. Pour Tenenbaum (2000, p. 22), «l'accès à la grand-parentalité conduit à une position identificatoire double, symétrique, à la fois à l'enfant qu'on a été et à son propre aïeul disparu ».

L'accès à une grand-maternité «maternelle» représente un enjeu psychique spécifique. Le report de la maternité d'une femme à une autre entre en jeu : «Le rapport mère-fille est placé sous le registre de l'indifférenciation possible, de la tentation de l'indifférenciation, du fantasme latent de corps-à-corps » (Bouchet, 1992, p. 11). La grand-maternité dans la lignée féminine constitue une crise narcissique et objectale importante dans le sens où « la maternité de la fille comme "événement" à la fois imaginaire et réel vient mettre en péril l'organisation psychique de sa mère » (ibid., p. 143). L'utilisation du mot «péril » nous interroge et nous évoque les enjeux liés à la dette de vie (Bydlowski, 1997). Les difficultés de remaniement psychique sont notamment à même de générer des infertilités psychogènes pouvant être comprises comme l'impossibilité pour certaines filles d'élire leur propre mère comme grandmère (Desjardins-Simon et Debras, 2010). Nous comprenons qu'il s'agit d'une étape délicate à franchir tant du côté de la fille que de la mère. La clinique montre de multiples destins de la grand-maternité : les liens peuvent se renforcer ou se distendre. Dans tous les cas, les remaniements identitaires chez les grands-parents sont incontournables. La mère devenue grand-mère par sa fille s'inscrit alors dans une forme de maternité symbolique par «fille interposée » où les notions de filiation sont profondément réactualisées (Kaës, 2009). Ces différents éléments révèlent que la vie psychique du sujet devenu grand-mère nécessite une investigation approfondie pour appréhender l'intensité des bouleversements psychiques inhérents. À notre connaissance, il n'existe aucuns travaux à ce sujet en appui sur la méthodologie Rorschach.

\section{Réaménagements psychiques du devenir grand-mère et expression somatique}

Toute relation familiale passant par le corps-à-corps (Baudin, 2012), expression somatique et réaménagements psychiques dans l'accès à la grand-maternité peuvent être étroitement liés. Un débordement pulsionnel, provoqué par la nécessité de réaménagements intrapsychiques et intersubjectifs au sein de la famille, peut en effet venir s'exprimer dans et par le corps.

Dans le modèle de la psychosomatique de l'École de Paris (Marty, M’Uzan et David, 1963), l'expression somatique fait partie du déroulement normal de la vie de tout individu et 
constitue un mode de régulation au même titre que les régulations psychiques, mais aussi comportementales, caractérielles ou par les investissements extérieurs (Debray, 2001, 2009). L'expression somatique traduit une difficulté à traiter sur le plan mental des excitations et revêt donc une fonction économique (Marty, 1990), voire de protection de l'appareil psychique : c'est le corps et non pas le psychisme qui subit la désorganisation (Dejours, 2009). En ce sens, le symptôme n'a de valeur que s'il est rapporté à l'économie psychosomatique générale du sujet. En effet, si le symptôme peut être le signe d'une déliaison pulsionnelle ou d'une régression psychique (Smadja, 1998), il peut aussi avoir une valeur positive en constituant une étape vers le processus de symbolisation (Dejours, 2009). De ce fait, " un même trouble somatique peut avoir une valeur différente selon les individus, mais aussi chez le même individu selon les moments de sa vie » (Debray, 2009, p. 23). En ce sens, la période de l'accès à la grand-maternité correspond à un moment de vie particulier qui mérite d'être pris en compte dans l'étude du fonctionnement mental d'un sujet présentant une affection somatique.

Notre problématique de travail a donc été double : quels peuvent être les réaménagements psychiques d'un sujet confronté au devenir grand-parent ? Et comment peut se comprendre une expression somatique dans ce contexte précis ? Nous faisons l'hypothèse que l'accès à la grand-maternité reconvoque les relations du sujet à ses imagos parentales et grand-parentales et engage des bouleversements psychiques denses qui peuvent notamment se traduire dans la sphère somatique par l'apparition d'une symptomatologie d'appoint transitoire, comme nous proposons de l'illustrer avec la situation de Catherine.

\section{Présentation de l'étude dont est extraite la situation clinique}

La situation clinique présentée dans cet article est issue d'une recherche conduite au centre hospitalier régional universitaire de Besançon de mai 2016 à avril 2018 auprès de sujets migraineux. Il était proposé à chaque sujet consultant pour la première fois dans le service de neurologie de participer à notre étude longitudinale. Le protocole de recherche prévoyait deux temps d'inclusion : avant le début de la prise en charge médicale (T1) et six à neuf mois plus tard (T2), une fois la symptomatologie apaisée par le traitement médicamenteux prescrit par le neurologue lors de la première consultation. Dans cette étude, la passation de l'épreuve projective Rorschach était prévu T1 et au T2 de l'étude, ceci afin d'apprécier l'évolution psychique du sujet. Malgré l'intérêt qu'aurait pu présenter le recours au TAT comme épreuve complémentaire ${ }^{1}$, nous avons fait le choix de ne pas inclure cette épreuve dans notre protocole dans la mesure où celui-ci prévoyait par ailleurs déjà de présenter à chaque patient un certain nombre d'outils (échelle d'évaluation de l'activité de la maladie, auto-questionnaires, test neuropsychologique) nécessitant pour le $\mathrm{T} 1$ deux rencontres de une heure et demi à deux heures chacune (pour le détail de l'étude, voir Bouteloup, 2020).

La situation de Catherine nous a paru intéressante à présenter car, contrairement à la grande majorité des patients migraineux rencontrés, la symptomatologie est récente. Cette patiente, devenue grand-mère au $\mathrm{T} 2$ de notre recherche, répond très favorablement à la prise en charge puisque cette symptomatologie a pratiquement disparu au moment du suivi. La dimension longitudinale de notre étude nous permet alors d'illustrer le parcours individuel d'un sujet devenu grand-mère, notamment à travers l'étude de deux protocoles Rorschach selon les principes de l'École de Paris (Chabert, 1983).

\footnotetext{
${ }^{1}$ Pour l'approche des modalités identificatoires et d'inscription générationnelle.
} 
En effet, le Rorschach apparait comme un outil privilégié pour apprécier le fonctionnement psychique du sujet et son évolution, et ce à tous les âges de la vie (Chabert, 1983 ; Péruchon, 2005). Dans la clinique du vieillissement, les méthodes projectives ont démontré leur intérêt pour repérer la particularité des fonctionnements psychiques à cette période de la vie (Péruchon, 2002 ; Verdon, 2017). De même, dans la clinique psychosomatique, le Rorschach a également démontré sa validité et sa pertinence (Debray et Belot, 2008 ; Pheulpin, Baudin et Bruguière, 2017). Enfin, des travaux sur le devenir mère ont permis de mettre en avant que, hors contexte psychopathologique, le fonctionnement psychique au Rorschach des femmes en post-partum présentait des particularités révélant notamment des modifications sur le plan narcissique et objectal (Belot, 2014). En ce sens, pour l'étude des protocoles Rorschach de Catherine, nous avons fait le choix de centrer notre analyse sur les planches considérées dans la littérature comme évocatrices des relations précoces et/ou des imagos maternelles et paternelles (Chabert, 1983) afin d'étudier plus précisément les réorganisations objectales en lien avec le devenir grand-mère.

\section{Situation clinique}

Catherine est une femme d'une cinquantaine d'années occupant un poste à responsabilités dans une grande entreprise. Elle est mariée et a deux enfants adultes. Femme coquette, grande et de forte corpulence, Catherine s'exprime de façon posée et essaie d'adopter un registre langagier recherché et parfois soutenu. Elle prend plaisir à venir aux rendez-vous et à expérimenter la rencontre avec un psychologue.

\section{Situation somatique à l'entrée dans le protocole de recherche}

Catherine présente un bon état de santé général mais une symptomatologie migraineuse s'est installée seulement quelques mois avant notre rencontre. Catherine souffre environ une fois par semaine de troubles visuels (vision en caléidoscope), de troubles de l'élocution (impossibilité d'exprimer sa pensée et de trouver ses mots) et de paresthésies (fourmis dans les mains) suivis de violents maux de tête de plusieurs heures nécessitant un arrêt de toute activité et dont elle évalue la douleur à 8 sur 10. L'intensité des symptômes migraineux a nécessité une investigation médicale approfondie. Une IRM a révélé la présence d'un anévrisme bénin qui ne pouvait aucunement expliquer les symptômes rapportés et qui ne représente a priori aucun danger. Cette découverte a cependant été source de fortes angoisses pour Catherine dont une amie est décédée d'une rupture d'anévrisme à l'âge actuel de notre patiente : «J'ai pensé à cette amie qui est morte, sa fille attendait un bébé et elle n'a même pas vu son petit-fils et je me suis dit ça y est, c'est mon tour. Je me voyais comme elle. Et puis j'ai dit à ma fille, avant de mourir j'aurais tellement aimé être grand-mère. »

Pour prévenir les crises migraineuses, Catherine a pris l'habitude de consommer plusieurs cachets d'antidouleur par jour. Cette surconsommation de médicaments a généré des céphalées chroniques quotidiennes: en plus des crises migraineuses hebdomadaires, des douleurs quasi-quotidiennes mais de faible intensité (évaluées à 3 sur 10) et sans symptôme associé se sont installées. Le neurologue prodigue à Catherine les conseils nécessaires pour l'arrêt de cette consommation abusive et lui prescrit un traitement de fond pour réduire les crises migraineuses. Les éléments qui suivent sont issus des entretiens de recherche.

\section{Anamnèse}


Catherine est l'aînée d'une fratrie de trois enfants. Ses parents ont été très investis dans leur propre vie professionnelle, avec des horaires décalés, et donc peu disponibles pour leurs enfants. La mère de Catherine, décrite comme une femme hyperactive, était cependant très attentive à l'organisation du quotidien pour ses enfants, préparant à l'avance les repas et assurant les tâches domestiques. Elle a également cherché à compenser par de nombreux cadeaux et une générosité financière son absence, dont Catherine dit avoir malgré tout souffert.

Catherine a cependant pu compter sur la présence de sa grand-mère, véritable confidente qui compensait le manque de ses parents : «J'ai abordé les questions de la vie avec ma grandmère. Si j'avais un problème c'est vers elle que j'allais en premier. » Catherine décrit une ambiance familiale construite sur la retenue et la rigueur. "J'ai été éduquée à toujours devoir montrer le meilleur de moi-même: le paraître, le savoir-être, pas de faille, c'est ce qui comptait. Chez nous on ne marquait jamais l'émotion et on ne montrait que le meilleur de soimême. »

\section{Vie actuelle}

Catherine est une femme très active : «J'ai un peu pris ça de maman, moi je ne sais pas trop me poser, il faut que je sois en permanence en train de faire quelque chose. » D'une manière générale, Catherine constate avoir des difficultés à exprimer ses sentiments, notamment avec ses enfants : «Bien sûr, quand ils étaient petits, c'étaient des bisous tout ça, mais quand ils ont grandi, y a une certaine distance qui s'est faite. Je voudrais bien être plus ouverte, arriver à faire valoir mes sentiments, mais je n'y arrive pas. »

Avec ses enfants, les relations sont de bonne qualité mais de factures très différentes. Catherine se décrit comme une mère poule, ce que son fils apprécie tout particulièrement : ne vivant plus au domicile parental, il continue à profiter des petits soins de Catherine qui s'occupe de son linge et lui prépare des Tupperware à remporter chez lui. A contrario, sa fille a ressenti le besoin de mettre de la distance avec sa mère, la trouvant «trop étouffante » : «C'est vrai que je vivais tout ce que vivait ma fille par procuration : quand elle était triste, j'étais aussi triste qu'elle. » Les relations avec son mari sont décrites comme de très bonne qualité. Catherine dit être satisfaite du soutien qu'elle reçoit de sa part, qui a d'ailleurs été présent à tous les rendez-vous avec les professionnels de santé.

Catherine fait un bilan très positif de sa vie, mettant notamment en avant sa réussite professionnelle, conjugale et financière. Elle repère un seul point négatif, mais très important pour elle : elle n'est pas encore grand-mère. Ceci représente une grande souffrance pour Catherine, qui reconnaît être jalouse de ses amies qui ont déjà des petits-enfants. Cependant, de nouvelles perspectives heureuses s'offrent à elle puisqu'au moment de notre rencontre elle vient d'apprendre la grossesse de sa fille. Cette naissance à venir représente pour Catherine une opportunité pour réussir à communiquer ses sentiments : "Je voudrais bien être un peu plus ouverte, réussir à ouvrir mon cœur. J'espère pouvoir le faire avec mon petit-fils et je suis sûre que ma fille va dire : "T'as pas été câline comme ça avec moi”. »

\section{À la sortie du protocole}

Nous revoyons Catherine sept mois après son entrée dans le protocole. La symptomatologie s'est nettement améliorée puisque Catherine n'a présenté que quatre crises de courte durée depuis l'instauration du traitement de fond. Sur le plan personnel, Catherine dit être 
transformée depuis la naissance de son petit-fils. Elle prend énormément de plaisir à nous montrer des photos sur son téléphone portable et nous raconte en détail son nouveau quotidien de grand-mère. Elle reste cependant toujours aussi investie dans son travail, mais envisage de poser ses RTT pour avoir plus de temps avec son petit-fils, chose qu'elle ne s'est jamais autorisée à faire depuis qu'elle est en poste. Toutes ses craintes autour de la difficulté à verbaliser ses sentiments ont disparu: Catherine se décrit comme «gaga» de son petit-fils âgé d'à peine un mois. Elle exprime se sentir enfin «normale, comme tout le monde » depuis qu'elle est devenue grand-mère et ne voit plus de point négatif dans sa vie.

\section{Résultats au Rorschach : extraits des planches ${ }^{2}$}

Nous avons fait le choix, comme exposé plus haut, de centrer notre propos sur l'analyse des planches où des symbolisations maternelles et/ou paternelles sont évoquées (pl. I ; IV ; VII ; IX ; X).

\begin{tabular}{|c|c|}
\hline \multicolumn{2}{|c|}{ Planche I } \\
\hline T1 & T2 \\
\hline $\begin{array}{l}\text { Réponse: } \\
\text { Alors comme ça franchement, ça me fait } \\
\text { penser à une matrice féminine, c'est-à-dire } \\
\text { un utérus }(G) \text {. Je peux difficilement penser à } \\
\text { autre chose. C'est la première pensée qui } \\
\text { est importante? } \\
\text { Alors une chauve-souris qui déploie ses } \\
\text { ailes. Ça m'inspire pas plus que ça. }\end{array}$ & $\begin{array}{l}\text { Réponse : } \\
\text { Je me rappelle pas du tout de ce que j'ai dit } \\
\text { la première fois! Alors là moi je vois une } \\
\text { chauve-souris qui a déployé ses ailes. } \\
\text { (?) Non pas plus. }\end{array}$ \\
\hline
\end{tabular}

$\mathrm{Au} \mathrm{T} 1$, Catherine fournit une première réponse anatomique très crue (utérus), révélant ici une difficulté à symboliser le maternel. Nous proposons de mettre cette réponse en lien avec la grossesse de sa fille qu'elle vient d'apprendre. L'évocation d'une matrice féminine traduirait ici une réactualisation en lien avec son propre vécu de femme enceinte et des préoccupations corporelles propres à la période anténatale. Il est en effet très fréquent de retrouver ce type de réponse chez les femmes enceintes dans la population tout-venant (Minjollet, 2017). Au T2, nous observons que ces préoccupations corporelles et les enjeux autour du fantasme de maternité se sont éteints, ce qui peut traduire le travail d'élaboration entre le T1 et le T2 autour du féminin/maternel. Le refoulement apparaît comme opérant et de bonne facture puisque ne subsiste que la réponse «chauve-souris », déjà présente en deuxième réponse au T1.

\begin{tabular}{|l|l|}
\hline \multicolumn{1}{|c|}{ Planche IV } \\
\hline \multicolumn{1}{|c|}{ T1 } & \multicolumn{1}{|c|}{ T2 } \\
\hline Réponse: & Réponse : \\
C'est plus compliqué. Je verrais un arbre, & Ah bah là pour moi c'est un arbre, ça \\
un chêne très majestueux, solide, avec un & représente un chêne, le tronc et tout le \\
tronc large, quelque chose d'imposant. Le & feuillage majestueux et tout. \\
maître de la nature. &
\end{tabular}

\footnotetext{
${ }^{2}$ L'intégralité des planches et le détail de l'analyse des protocoles Rorschach, qui n'ont pu être insérés ici du fait de contraintes éditoriales, peuvent être fournis sur simple demande par mail aux auteurs.
} 
Catherine évoque spontanément une représentation caractérisée par la massivité et la puissance phallique traduisant de bonnes capacités de symbolisation à cette planche. La thématique de la fécondité est une nouvelle fois présente. Cette réponse est stable entre le T1 et le T2 mais révèle une certaine évolution psychique. Alors qu'au T1 l'accent est mis sur un tronc solide, symbole de l'ancrage dans le sol pouvant (sup)porter une lignée familiale, au T2 l'apparition du feuillage «majestueux » dans la réponse révèle l'apparition de la filiation et l'apport narcissique des nouvelles générations pour l'ensemble familial représenté par l'arbre, nous évoquant «His Majesty The Baby» (Freud, 1914).

\begin{tabular}{|l|l|}
\hline \multicolumn{2}{|c|}{ Planche VII } \\
\hline \multicolumn{1}{|c|}{ T1 } & \multicolumn{1}{|c|}{ T2 } \\
\hline Réponse : & Réponse : \\
Ça me semble, on... de prime abord, comme & Je verrais plutôt un continent, la mer qui \\
ça, pour moi c'est un paysage au bord de la & rentre dans les terres et ça c'est des roches, \\
mer. Pour moi j'ai eu un flash, j'ai vu un & ça fait comme un bassin touristique, \\
port d'une région que j'aime bien, on dirait & méditerranée et tout, ouais. \\
qu'on le voit depuis le haut, depuis une & \\
falaise, avec la mer qui s'engouffre dans le & \\
port. & \\
\hline
\end{tabular}

À la planche VII, la réponse au T1 fait référence à la dimension féminine et maternelle par le creux. Le symbolisme sexuel est présent. Le maternel féminin est représenté par la baie dans laquelle la mer s'engouffre. Ce type de réponse évoque là encore le féminin maternel symbolisé mais de facture archaïque en lien avec la conception. La réponse est identique à T2 mais moins idéalisée. La secondarisation est plus importante et la notion de «bassin » est déplacée sur un contenu géographique marquant la mise à distance par l'intellectualisation et l'extinction du fantasme d'être enceinte.

\begin{tabular}{|l|l|}
\hline \multicolumn{4}{|c|}{ Planche IX } \\
\hline \multicolumn{4}{|c|}{ T1 } & \multicolumn{1}{|c|}{ T2 } \\
\hline Réponse : & Réponse : \\
Alors... Là y a rien qui me vient & Qu'est-ce que ça peut... \\
franchement... Non là j'ai aucune & Moi je verrais un vase ou une sculpture avec \\
inspiration, aucun flash. Ça peut arriver ? & une partie centrale en verre transparent, ça \\
Je vais pas broder ou dire n'importe quoi & serait plutôt une cuvre d'art, quelque chose \\
hein, ça veut rien dire. & qui pourrait recueillir des fleurs. \\
\hline
\end{tabular}

$\mathrm{Au} \mathrm{T1}$, le refus traduit un mouvement de sidération psychique du fait de l'intensité de la sollicitation pulsionnelle à cette planche reconnue comme «utérine » (Chabert, 1983). Notre hypothèse est celle d'un fonctionnement psychique saturé par l'intensité de la réactualisation de fantasmes liés au féminin et au maternel. Au T2, alors que Catherine est devenue grandmère, les difficultés d'élaboration ne sont plus présentes. Elle fournit une réponse marquant une symbolisation de qualité du maternel contenant et de la réceptivité féminine. Il s'agit ici pour nous d'un remaniement psychique pulsionnel conséquent.

\begin{tabular}{|c|c|}
\hline \multicolumn{2}{|c|}{ Planche X } \\
\hline T1 & T2 \\
\hline
\end{tabular}




\begin{tabular}{|l|l|}
\hline Réponse: & Réponse : \\
Ah ben là c'est plus jovial, c'est plus une & Ya beaucoup de choses... \\
ouverture vers l'extérieur, il y a plus de & Alors, c'est peut-être totalement loufoque \\
couleurs. On dirait la fête, des feux & mais je verrais la tour Eiffel avec les jardins \\
d'artifice ou des confettis, une situation & de la tour Eiffel, avec le Champ de Mars et \\
joviale. Ouais, un feu d'artifice. & tout, c'est cette impression-là. \\
\hline
\end{tabular}

La réponse "feu d'artifice » au T1 révèle la mise en place d'un mécanisme de défense hypomane à valeur antidépressive. Aux sollicitations latentes morcellantes de cette planche X, Catherine parvient à unifier les parties pour en former un tout dans une représentation de fête et de jovialité. Au T2, Catherine fonctionne encore mieux sur le plan libidinal puisqu'apparaît une symbolisation phallique par l'évocation d'une «tour Eiffel» entourés de jardins ordonnés. Mais si la tour Eiffel évoque un symbole phallique (pointe de la tour), elle contient également un symbole maternel et protecteur par son assise et le contenant qu'elle offre à sa base. L'accès à la grand-parentalité semble ainsi modifier considérablement le paysage familial avec l'évocation d'un champ de Mars, vecteur de puissance.

\section{Discussion}

\section{La disparition de la symptomatologie migraineuse}

Rappelons que Catherine a été rencontrée dans le cadre d'une recherche auprès de patients migraineux. Alors qu'au T1 la symptomatologie migraineuse est chronique et invalidante, les crises ont pratiquement disparu au $\mathrm{T} 2$, signe d'une évolution particulièrement positive ${ }^{3}$. La symptomatologie pourrait ainsi témoigner d'une fonction d'écoulement des excitations non psychéisables et en ce sens a pu constituer une symptomatologie d'appoint (Debray, 2001).

L'apparition des migraines à l'âge exact où sa meilleure amie, sur le point de devenir grandmère, est décédée d'une rupture d'anévrisme pourrait également être le signe de préoccupations corporelles inconscientes de Catherine au sujet de son propre devenir grandmère. En effet, selon les données de l'entretien clinique, la découverte d'un anévrisme bénin à ce moment précis a généré des angoisses mortifères et a conduit Catherine à une prise de conscience de ses états internes. Elle parvient à verbaliser à sa fille non seulement ses craintes de mourir mais aussi sa déception de ne pas être grand-mère, alors même que Catherine a été élevée dans une certaine forme de retenue émotionnelle qu'elle a reproduite dans ses rapports avec ses enfants. - encore aujourd'hui, elle a prioritairement recours aux actes pour leur manifester son attachement. Les éléments de l'entretien clinique ont montré que Catherine s'autorise désormais à mettre en mots des ressentis qui étaient jusqu'alors réprimés. Ceci illustre la façon dont l'appareil psychique peut être mis au travail suite à l'apparition d'une symptomatologie corporelle. En ce sens, le symptôme peut avoir une valeur de somatisation symbolisante au sens de Christophe Dejours (2009).

De même, nous questionnons l'apparition de cette symptomatologie très spécifique, associée dans le sens commun à un refus de la sexualité dans le lit conjugal, dans une période où le corps féminin et la vie sexuelle se transforment du fait de la ménopause : les migraines de Catherine viennent-elles représenter un symptôme signant une forme de castration de sa femelléité (Chiland, 1991) afin de signifier à sa fille qu'elle lui « laisse la place » de devenir mère ? Monique Bydlowski (1997) évoque en effet que, pour accéder à la maternité, la fille

\footnotetext{
${ }^{3}$ Ce type d'évolution très positive est relativement rare : le traitement permet généralement de réduire la symptomatologie, au moins dans son intensité, mais ne conduit presque jamais à une quasi-disparition des crises.
} 
doit pouvoir vivre sa propre mère comme «faible, vaincue, perdante, aimée parce que faible ». La découverte d'un anévrisme et les symptômes migraineux peuvent en ce sens avoir été « au service » de cette dynamique mère-fille.

\section{La grand-maternité : une possibilité de trouver sa place dans la lignée familiale}

L'étude des réponses aux planches IV et $\mathrm{X}$ du Rorschach illustre l'évolution de la structuration des générations dans le psychisme de Catherine et la valence narcissique que représente l'arrivée d'un petit-enfant. Les réponses au T2 peuvent en effet évoquer une filiation qui se dessine et qui donne consistance à l'ensemble familial qui se hiérarchiserait autour du petit-fils. Catherine peut alors trouver sa place dans la lignée familiale : «Plus que la maternité, c'est la grand-maternité qui forgerait le sens de la filiation » (Bouchet, 1992, p. 58).

Catherine souhaite aujourd'hui se détacher de l'attitude de réserve dans laquelle elle a grandi et donner à sa famille une autre tonalité affective que celle de l'ambiance familiale transmise par ses propres parents (au Rorschach, les thèmes de fête et de jovialité peuvent le refléter et en être l'expression). La possibilité d'une évolution au plan psychique ne peut alors passer que par l'investissement d'un petit-enfant, qui légitimerait une attitude chaleureuse et affectueuse et valoriserait son statut de grand-mère. Catherine semble ainsi se détacher progressivement de son rôle «d'enfant de ses parent» et attend de pouvoir prendre une position d'aïeule pour s'autoriser à agir comme sa grand-mère. Les relations de très bonne qualité avec sa propre grand-mère peuvent lui permettre de se projeter plus favorablement et avec sérénité mais aussi grand confort interne dans son rôle grand-maternel. En ce sens, le travail de remaniement identificatoire dans l'accès à la grand-maternité ne représente pas une difficulté pour Catherine, qui a la possibilité de s'identifier à une figure grand-maternelle beaucoup plus contenante et sécurisante que la figure maternelle.

\section{Le symbolique maternel réactualisé et psychiquement au travail}

Le Rorschach nous permet de relever un traitement différent des planches à symbolisation maternelle et féminine entre le T1 et le T2. Tout d'abord, l'appréhension de la planche I révèle une évolution significative : alors que l'identification à la grossesse de sa fille est majeure au T1, avec l'évocation d'une matrice féminine rappelant les fantasmes de maternité, le refoulement opérant au T2 traduit une évolution positive de Catherine dans son rapport au symbolique maternel. Les suggestions des planches autour du féminin-contenant sont également traitées aux planches VII et IX du T2, alors que la symbolisation n'apparait qu'à la planche VII au T1. Au T2, le travail psychique autour de l'imago maternelle apparaît davantage symbolisé et surtout à bonne distance, comme si l'accès à une posture grandparentale avait offert la possibilité à Catherine d'investir le féminin maternel dans sa dimension contenante et sécurisante. Ceci est probablement sous-tendu par l'identification positive et étayante de Catherine à son imago grand-maternelle qui a agi en substitut maternel. Le saut des générations et la construction d'un lien grand-mère/petit-enfant permet alors une certaine forme de réparation du féminin, voire de la représentation de son imago maternelle.

\section{Conclusion}

L'étude de la situation de Catherine nous permet d'illustrer les mouvements psychiques au Rorschach d'un sujet devenu grand-parent entre les deux passations. Pour Catherine, l'accès à la grand-maternité semble représenter une opportunité d'évoluer positivement dans son 
rapport à elle-même et aux autres. Elle aborde cette étape de vie avec tranquillité et même impatience, forte de nouvelles représentations de l'image maternelle liée au fonctionnement grand-parental qui agit comme substitut parental. Les modèles identificatoires grandmaternels sont sécurisants et guident Catherine dans l'accès à la grand-maternité.

Aussi, cette étude de cas donne à penser différents enjeux :

- la nécessité de s'intéresser davantage aux processus psychiques impliqués dans l'accès à la grand-parentalité car ils peuvent être une source de remaniements conséquents et positifs ;

- l'importance d'investiguer les remaniements intrapsychiques et interpersonnels que la grand-parentalité convoque. L'accès à la grand-maternité, tout comme le devenir mère, représenterait selon notre hypothèse une véritable crise développementale à l'image de ce qui a déjà été décrit pour l'accès à la maternité (Bibring, 1959). Cet état de crise, tout comme celui présent lors de l'accès à la maternité, aboutit à de nouvelles configurations psychiques qu'il est important de repérer ;

- la nécessité de prendre en compte l'environnement du sujet et les modifications internes qu'il impulse dans une approche phénoménologique : en effet, nous interrogeons les facteurs ayant participé à l'évolution positive de la symptomatologie de Catherine entre le T1 et le T2. Si le traitement médicamenteux peut agir bien entendu favorablement, nous avons relevé, grâce à l'analyse fine de l'épreuve projective Rorschach entre les deux temps, la présence de remaniements internes denses pouvant être liés à l'accès à la grand-parentalité. Seule une étude à plus grande échelle nous permettrait d'apporter des éléments de réponse à nos interrogations pour mieux appréhender les modifications intrapsychiques et psychosomatiques liées à l'accès à la grand-parentalité.

\section{Bibliographie}

Attias-Donfus, C. 2008. «Les grands-parents en Europe : De nouveaux soutiens de famille », Informations sociales, 5,149, 54-67.

Baudin, M. 2012. "Missions et transmissions : Aux sources de quelques énigmes du corps », Dialogue, 197, 3, 7-17.

Belot, R.-A 2014. «La singularité des protocoles Rorschach chez une population de femmes en période postnatale », Devenir, vol. 26, 3, 165-204.

Belot, R. ; Vennat, D. ; Moissenet, A. ; Bluon-Vannier, A. ; Herse, V. ; Montigny (de), F. ; Lacharité, C. ; Mellier, D. 2013. «Accès à la parentalité et isolement familial. La nouvelle solitude des parents », Dialogue, 199, 1, 7-18.

Bibring, G.L. 1959. "Some considerations of the psychological processes in pregnancy », Psychoanal. Study Child, 14, 113-121.

Bouchet, S. 1992. De mère à grand-mère : Approche psychanalytique d'une identité nouvelle, Paris, Bayard.

Bouteloup, M. 2020. "Céphalées primaires et variables individuelles : attachement, mentalisation et économie psychosomatique », Bulletin de psychologie, 73,1, 69-72.

Bydlowski, M.1997. La dette de vie. Itinéraire psychanalytique de la maternité, Paris, Puf, 2008.

Chabert, C. 1983. Le Rorschach en clinique adulte : interprétation psychanalytique, Paris, Dunod, 2012.

Chiland, C. 1991. «Castration et féminité », dans A. Le Guen (sous la direction de), Angoisse et complexe de castration, Paris, Puf, 81-92.

Debray, R. 2001. Épître à ceux qui somatisent, Paris, Puf.

Debray, R. 2009. «Psychopathologie et somatisation», dans C. Chabert (sous la direction 
de), Psychopathologie des limites : Traité de psychopathologie de l'adulte, Paris, Dunod, 351 .

Debray, R.; Belot, R.-A. 2008. La psychosomatique du bébé, Paris, Puf.

Dejours, C. 2009. Les dissidences du corps Paris, Payot \& Rivages.

Desjardins-Simon, J. ; Debras, S. 2010. Les verrous inconscients de la fécondité, Paris, Albin Michel.

Freud, S. 1914. « Pour introduire le narcissisme », dans La vie sexuelle, Paris, Puf, 1977.

Kaës, R. 2009. «La réalité psychique du lien », Le divan familial, 22,1, 107-125.

Le Borgne-Uguen, F. 2003. "Grands-parents : un rôle à composer. Un enjeu entre générations, une étape dans le parcours de vie », Empan, 52, 4, 77-85.

Lebovici, S. 1991. «Être grand-parent », Journal de pédiatrie et de puériculture, 3, 179-82.

Marty, P.1990. La psychosomatique de l'adulte, Paris, France, Puf, 2004.

Marty, P. ; M'Uzan (de), M. ; David, C. 1963. L’investigation psychosomatique : sept observations cliniques, Paris, Puf.

Mietkiewicz, M. ; Schneider, B. 2010. « Devenir vieux en famille : les mamies et les papys de la littérature jeunesse », Dialogue, 188, 2, 83-98.

Mietkiewicz, M. ; Schneider, B. 2013. «Entre l'enfant et ses grands-parents, une bonne distance en construction », $6^{\mathrm{e}}$ colloque du RIPSYDEVE, Actualités de la psychologie du développement et de l'éducation, 161-167.

Miljkovitch, R. 2005. «Entre grands-parents et petits-enfants. L'impact des grands-parents sur les représentations d'attachement de leurs petits-enfants », dans S. Bouyer, M.-C. Mietkiewicz et B. Schneider (sous la direction de), Grands-parents et grands-parentalités, Toulouse, érès, 77-99.

Minjollet, P. 2017. Quand la grossesse prend corps : de la grossesse à un an postpartum, approche clinique et projective de l'image du corps de la femme en période périnatale, Thèse de doctorat, [en ligne] http://www.theses.fr/2017USPCC086.

Péruchon, M. 2002. "La névrose dans le grand âge à l'appui d'épreuves projectives », Cahiers de psychologie clinique, 18, 1, 45-56.

Péruchon, M. 2005. «Grandir, mûrir, vieillir. Introduction au colloque de la Société française $\mathrm{du}$ Rorschach et des méthodes projectives », Psychologie clinique et projective, 11, 1, 223230.

Pheulpin, M.-C.; Baudin, M. ; Bruguière, P. 2017. Cliniques des états du corps : Approche psychanalytique et projective, Paris, Dunod.

Schneider, B. ; Mietkiewicz, M. ; Bouyer, S. 2005. «Introduction», dans S. Bouyer, M.C. Mietkiewicz et B. Schneider (sous la direction de), Grands-parents et grands-parentalités, Toulouse, érès, 7-26.

Smadja, C. 1998. «Le fonctionnement opératoire dans la pratique psychosomatique », Revue française de psychanalyse, 62, 5, 1367-1440.

Soulé, M. 1979. Les grands-parents dans la dynamique de l'enfant, Paris, ESF.

Tenenbaum, H. 2000. «Y a-t-il un art d'être grand-père... ou grand-mère ? », dans S. Bouyer, M.-C. Mietkiewicz et B. Schneider (sous la direction de), Histoire(s) de grands-parents, Paris, L'Harmattan.

Thévenot, A. 2005. " "On commence à former une famille..." Une modalité de la construction de la place de grand-parent : à travers le regard des nouveaux parents », dans S. Bouyer, M.-C. Mietkiewicz et B. Schneider (sous la direction de), Grands-parents et grands-parentalités, Toulouse, érès, 29-42.

Vennat, D. ; Panagiotou, D. ; Mellier, D. 2018. «Idéologies hypermodernes, quels enjeux dans la construction de la parentalité ? », Bulletin de psychologie, 556, 4, 749-757. 
Verdon, B. 2017. «La pratique des épreuves projectives dans la clinique du vieillissement», dans M. Emmanuelli (sous la direction de), L'interprétation des épreuves projectives : l'École de Paris, Toulouse, érès, 207-214. 